\title{
SH-SY5Y-derived neurons: A neuronal model system for investigating TAU sorting mechanisms and neuronal subtype-specific TAU vulnerability
}

Bell, Michael ${ }^{1,2}$, Zempel, Hans ${ }^{1,2}$

(Corresponding author: Michael Bell, michael.bell@uk-koeln.de)

1 Institute of Human Genetics, University Hospital Cologne, Kerpener Str. 34, 50931 Cologne, Germany.

2 Center for Molecular Medicine Cologne (CMMC), Robert-Koch-Str. 10, 50931 Cologne, Germany.

\section{Abstract}

The human-derived SH-SY5Y neuroblastoma cell line is widely used for studying TAU physiology and TAU-related pathology in Alzheimer's disease (AD) and related tauopathies. SH-SY5Y cells can be differentiated into neuron-like cells (SH-SY5Y-derived neurons), which resemble noradrenergic, dopaminergic or cholinergic neurons, by using various substances. This review evaluates whether SH-SY5Y-derived neurons are a suitable model for investigating intracellular TAU sorting mechanisms in general, and with respect to neuron subtype-specific TAU vulnerability. SH-SY5Y-derived neurons show pronounced axodendritic polarity resembling neuronal cell polarity, high TAU protein levels, axonal TAU localization, expression of the six major human brain isoforms, and TAU phosphorylation similar to AD. This enables studying the isoformand phosphorylation-dependent impact on TAU subcellular distribution and axodendritic trafficking of TAU. As SH-SY5Y cells are accessible for genetic engineering, stable transgene integration and leading-edge genome editing are valuable and often-used tools for TAU-related research in these cells. Furthermore, SH-SY5Y-derived neurons resemble cells of distinct subcortical nuclei, i.e. the Locus coeruleus (LC), Nucleus basalis (NB) and Substantia nigra (SN), depending on the used differentiation procedure. This allows to study neuron-specific TAU isoform expression and intracellular localization in the context of vulnerability to TAU pathology. Limitations are e.g. the lack of mimicking age-related tauopathy risk factors and the difficulty to define the exact neuronal subtype of SH-SY5Y-derived neurons. In brief, this review discusses the suitability of SH-SY5Yderived neurons for investigating TAU sorting mechanisms and neuron-specific TAU vulnerability in human-like conditions.

Key words: SH-SY5Y-derived neurons, TAU sorting, neuronal identity, tauopathy, Alzheimer's disease 


\section{Introduction}

Alzheimer's disease $(A D)$ and related tauopathies constitute a major scourge of modern society healthcare due to their tremendously high and increasing prevalence ${ }^{1}$. The microtubule (MT)associated protein TAU, which is abundant in axons of mature neurons, is a key player for the pathological cascade of these diseases. Missorting into the somatodendritic compartment, sitespecific hyperphosphorylation and formation of neurofibrillary tangles (NFT) containing TAU are classical hallmarks of $A D$ and other tauopathies ${ }^{2-4}$. In the last decades, a lot of scientific effort has been invested in unravelling the physiological functions and pathomechanisms connected to TAU. Often-used rodent models (primary neuron cultures, animal models) require animal resources and suffer from limitations in translatability, such as different isoform expression ratios, artificial genetic settings due to multiple human transgenes, or species-dependent deviations regarding the cellular machinery and interactions partners ${ }^{5}$. Human induced pluripotent stem cell (hiPSC)-derived neurons overcome many of the described obstacles and constitute a powerful tool for TAU-related research ${ }^{6-10}$. However, differentiation of hiPSC-derived neurons is expensive, time-consuming and results in cultures with variable homogeneity and differentiation efficiency 11,12 .

The neuroblastoma cell line SH-SY5Y, which was subcloned from the SK-N-SH line ${ }^{13}$, is an easyto-handle and proliferative cell line with well-established differentiation methods for stable neuronal cultures. SH-SY5Y-derived neurons have been widely used for TAU-related research, as they yield homogeneous, reproducible human-derived neuronal cultures with robust expression and axonal distribution of TAU, thereby suitable also for addressing axonal TAU sorting. Interestingly, the neuronal identity of SH-SY5Y-derived neurons depends on the used differentiation procedure (reviewed in ${ }^{14}$ ), which bears great potential for neuronal subtype-specific TAU research.

The current review aims to evaluate the general suitability of SH-SY5Y-derived neurons for research on mechanisms of TAU sorting, focusing on TAU cellular polarity, total TAU and TAU isoform expression, intracellular localization, phosphorylation, and the possibilities of genetic engineering in SH-SY5Y cells. Moreover, the chances and challenges of the procedure-dependent identity of SH-SY5Y-derived neurons will be discussed regarding their utility to mimic neuronal subtypes of brain regions that are early affected in AD. 


\section{General suitability of SH-SY5Y-derived neurons for TAU sorting research}

Neuronal polarity and total TAU expression. A suitable neuronal cell model for TAU sorting research needs to meet certain demands, including resemblance of neuronal morphology in form of pronounced neuronal polarity and axonal outgrowth, neuronal maturation, indicated by common neuronal marker proteins ${ }^{15}$ and, strikingly, high expression levels and axonal localization of TAU protein.

Little amounts of TAU protein are detectable in undifferentiated SH-SY5Y cells, in which it is present both in the cytoplasm and the nucleus ${ }^{16}$. Differentiation of SH-SY5Y cells, inducible with retinoic acid (RA) and various other substances (reviewed in ${ }^{14}$ ), results in neurite outgrowth and strong increase of overall TAU protein levels ${ }^{17,18}$ with a neuron-like subcellular distribution, i.e. increased axonal and decreased somatic TAU levels (Fig. 1A,B) ${ }^{16,17,19}$. The use of combinatorial treatments, e.g. RA and brain-derived neurotrophic factor (BDNF) ${ }^{17,19,20}$ or BDNF and neuronal growth factor ${ }^{17,19,20}$, further enhances the axonal outgrowth, the total TAU expression to levels comparable with the human brain ${ }^{19}$, and the segregation of axonally localized TAU and somatodendritically localized microtubule-associated protein 2 (MAP2) (Fig. 1B) ${ }^{17,20}$, indicating a pronounced neuronal maturity and polarity ${ }^{21}$. Moreover, differentiation leads to drastic upregulation of other common neuronal maturation markers in SH-SY5Y cells, such as synaptophysin, neuronal nuclei, neuron specific enolase, growth-associated protein or synaptic vesicle protein II ${ }^{17,19,22-26}$. Whether SH-SY5Y-derived neurons exhibit the formation of the axon initial segment (AIS), a key player in developing and maintaining cellular and TAU protein polarity 27-31, was not yet addressed. It remains unclear if a detectable AIS precedes or coincides the neuronal maturation in SH-SY5Y-derived neurons, as it is typically seen during neuronal development ${ }^{27}$.

The strong expression of TAU, but also the upregulation of other neuronal markers such as MAP2 indicate the neuronal character of SH-SY5Y-derived neurons and make them a valuable system for TAU sorting-related studies. In other words, the neuron-like TAU localization enables the investigation of TAU sorting mechanisms in these cells. In this context, it would be worth to examine if SH-SY5Y-derived neurons properly distribute also transfected TAU, an often-faced challenge when working with neuronal cultures ${ }^{32}$. Given the formation of an AIS in fully 
differentiated cells, which needs to be evaluated in future studies, this model could also serve for the investigation of TAU-AIS interactions that are responsible for the regulation of retrograde and anterograde TAU trafficking, which takes place at the AIS ${ }^{28,30,31}$. Especially the question of anterograde TAU transit at the proximal axon is striking as the lack of stable microtubule structures within this compartment ${ }^{33}$ make continuous MT-directed transport unlikely.

TAU isoform expression. Alternative splicing of the exons 2 and 3 (either $0 \mathrm{~N}$, $1 \mathrm{~N}$, or $2 \mathrm{~N}$ isoforms) as well as exon 10 (3R or 4R isoforms) results in six major TAU isoforms in the mature human brain ${ }^{34-36}$. The isoform expression pattern is brain region-specific and changes during developmental stages ${ }^{34,37-39}$. The axodendritic distribution is markedly different depending on the TAU isoform ${ }^{31}$. Therefore, a suitable model for studying TAU sorting should display the expression of all human TAU isoforms.

Early studies investigating the TAU isoform expression showed consistently that undifferentiated SH-SY5Y cells express only the shortest TAU isoform ON3R ${ }^{16,18,40,41}$. More recently, TAU mRNA containing exon $2(1 \mathrm{~N})$ or exon $10(4 \mathrm{R})$ isoforms was found in untreated SH-SY5Y cells, suggesting at least basal expression of larger isoforms (Fig. 1C) ${ }^{19}$. Reports about differentiated SH-SY5Y-derived neurons vary in their described isoform expression pattern. Former studies detected either no shift in isoform expression upon RA treatment ${ }^{18}$, weak expression of an additional $64 \mathrm{kDa}$-sized isoform (probably representing the $2 \mathrm{~N} 4 \mathrm{R}$ isoform) ${ }^{40}$, or low levels of $4 \mathrm{R}$ isoform mRNA upon three weeks of RA treatment ${ }^{16}$. More recent findings, however, showed that undifferentiated SH-SY5Y cells already express high amounts of $1 \mathrm{~N}$ isoforms, and that differentiated cells express all six isoforms found in the human brain (Fig. 1C) ${ }^{19}$.

The reported isoform ratio in SH-SY5Y-derived neurons differs notably from the human brain ${ }^{42}$, with more $3 R$ than $4 R$ TAU, less $2 \mathrm{~N}$ isoforms and more $0 \mathrm{~N} 3 \mathrm{R}-\mathrm{TAU}^{19}$. This may suggest that a cultivation time of up to three weeks produces $\mathrm{SH}$-SY5Y-derived neurons at an intermediate stage of maturity. Later studies, however, found roughly equal amounts of $3 R$ and $4 R$ isoforms, as typically seen in the adult human brain ${ }^{43-46}$, after RA treatment ${ }^{47}$. Despite this discrepancy regarding the isoform ratio, these studies demonstrate the abundant presence of all six TAU isoforms, which makes SH-SY5Y-derived neurons valuable for further investigation of TAU isoforms in the context of TAU distribution and mislocalization. One bottleneck of tracing the emergence and progression of $A D$ in recent mouse models is, despite harbouring human TAU transgenes, the presence of all six human isoforms. Although recent transgenic mouse lines achieve the expression of all six human isoforms in ratios similar to the human brain ${ }^{48,49}$, they 
inevitably lack a human cellular environment, in contrast to SH-SY5Y-derived neurons. In other words, SH-SY5Y-derived neurons can help to clarify if and how different TAU isoforms contribute to cellular TAU functions under physiological conditions and possibly convey tauopathy-related toxicity, e.g. by being more susceptible for mislocalization, hyperphosphorylation or aggregation. The fact that distinct tauopathies show a distinct composition of TAU-NFTs, e.g. consisting only of $3 \mathrm{R}$ or $4 \mathrm{R}$ isoforms or a combination of both ${ }^{46,50}$, strongly indicates a connection between isoform expression and aggregation propensity, which warrants further investigation. Taken together, the TAU isoform ratio of SH-SY5Y-derived neurons differs from that in the mature human brain, but the strong expression of all six major isoforms already upon brief differentiation periods allows investigating isoform-specific localization and mislocalization propensity.

TAU phosphorylation state. More than 90 reported phosphorylation sites illustrate the striking importance of these posttranslational modifications for TAU function ${ }^{46}$. The phosphorylation state of TAU directly influences the microtubule-binding affinity and thereby its mobility and intracellular localization ${ }^{51-55}$. Hyperphosphorylation of the AT8 motif (including Ser-202 \& Thr-205) or other motifs strongly correlates with somatodendritic missorting of TAU and is characteristic for NFTTAU formation ${ }^{55-61}$. Pseudo-phosphorylation of the four KXGS motifs was shown to cause TAU missorting and synapse loss ${ }^{30,62}$, hinting at the importance of TAU phosphorylation state and its regulation within the pathological cascade in $A D$ and related diseases.

Consequently, early TAU studies with SH-SY5Y cells put great effort into analysing the phosphorylation state of TAU in SH-SY5Y cells. They revealed that large portions of TAU residues, including Ser-199, Ser-202 (AT8 epitope), Thr-231/Ser-235 (AT180 epitope), Ser-262 (12E8 epitope) and Ser-396/Ser-404 (PHF1 epitope) are phosphorylated in undifferentiated SH-SY5Y cells (Fig. 1D) 18,41,63. As these residues largely overlap with those, which appear hyperphosphorylated in NFT-containing TAU, the TAU protein of SH-SY5Y cells was considered as abnormally phosphorylated in an AD-like manner ${ }^{41}$. However, the high degree of phosphorylation can be explained by the notably high levels of ON3R-TAU in undifferentiated $\mathrm{SH}$ SY5Y cells. In early developmental stages, when ON3R-TAU is the predominant isoform, TAU typically shows an increased phosphorylation level ${ }^{34,60}$. Interestingly, no substantial change in TAU phosphorylation was seen upon differentiation with RA ${ }^{18}$ despite the expression of larger TAU isoforms ${ }^{19,47}$. This might be, at least partially, due to the fact that 0N3R-TAUappears as the major isoform also in differentiating SH-SY5Y cells ${ }^{19}$. 
Okadaic acid and other phosphatase inhibitors were able to evoke AD-like TAU hyperphosphorylation, disassembly of microtubules and increased degeneration of undifferentiated SH-SY5Y cells $40,64,65$. This coincides with an inactivation of PP1 and PP2A phosphatases and an increased activity of MAPK, CDC2 and CDK5 kinases ${ }^{64}$, thereby confirming their regulating role for TAU modifications, as it was postulated from previous in vitro interaction assays (reviewed in ${ }^{46}$ ), and directly linking phosphorylation state, MT stability and cell death. In other words, SH-SY5Y cells - either undifferentiated or differentiated - turned out as a highly valuable system for addressing how TAU phosphorylation is modulated and how this modulation can be compromised in disease conditions. Thus, many recent TAU studies in SH-SY5Y cells focused on TAU(hyper)phosphorylation, including the role of kinases/phosphatases and cellular pathways in misbalancing the TAU phosphorylation state ${ }^{63,66-69}$, the influence of microgliamediated neuroinflammation on TAU expression levels and phosphorylation ${ }^{47,70-72}$, the effects of diabetes-associated hyperglycaemia on the phosphorylation state and TAU metabolism ${ }^{73-76}$, the connection of TAU phosphorylation and sleep disorders in AD patients ${ }^{77}$.

In brief, the phosphorylation state of TAU influences its subcellular distribution and plays an important role in the TAU mislocalization in disease conditions ${ }^{51-55}$. The TAU phosphorylation state in SH-SY5Y cells displays that of healthy human brains and even more that of AD patients 41,46, and its regulation involves known TAU-interacting kinases/phosphatases 64,66,67. This comparability makes SH-SY5Y cells not only a valid model for further investigation of TAU phosphorylation, but also provides options for examining the interplay of TAU phosphorylation and intracellular (mis)sorting. 

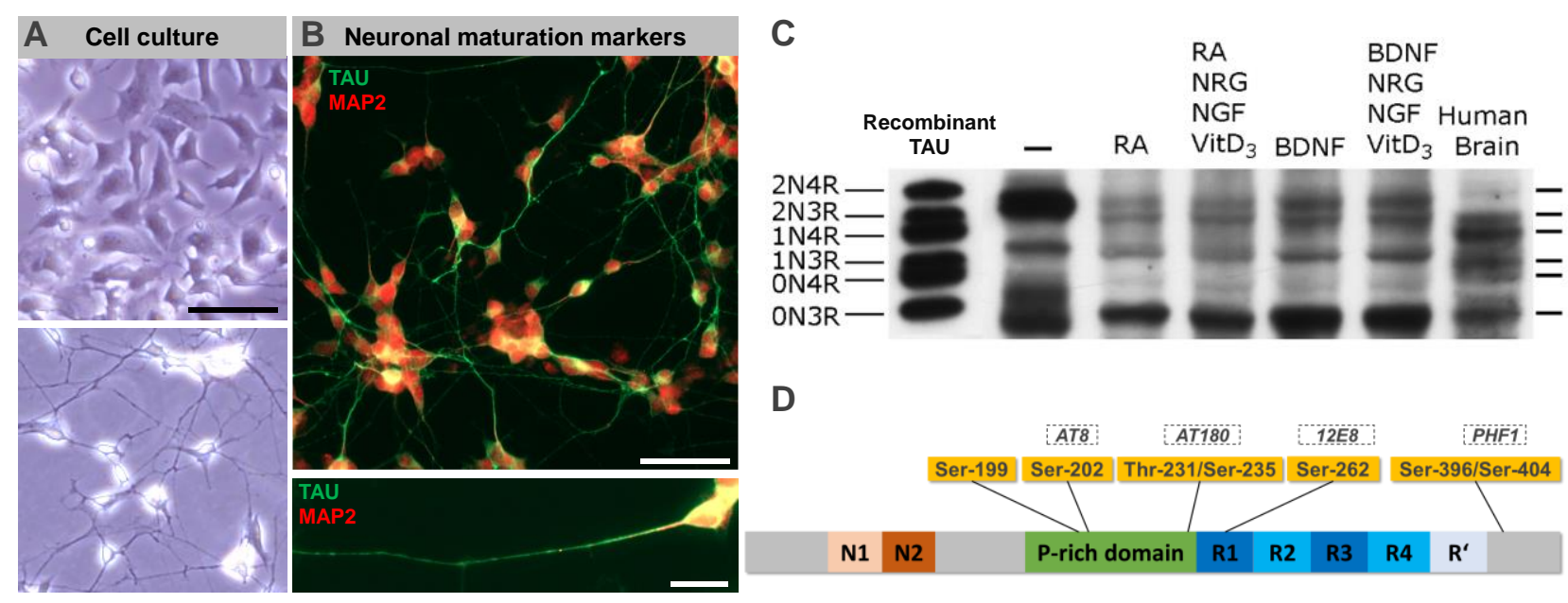

Figure 1: Suitability of SH-SY5Y-derived neurons for TAU sorting research. A) Representative images of undifferentiated SH-SY5Y cells (left, top panel) and SH-SY5Y-derived neurons (left, bottom) in culture (cultures were grown on Poly-D-Lysine $(20 \mu \mathrm{g} / \mathrm{ml})$-coated glass coverslips in DMEM/F12 (\#10565018, TFS) and $10 \%$ fetal bovine serum (BioChrom AG); for differentiation, cells were grown for 7 days in DMEM/F12, $10 \%$ fetal bovine serum and $10 \mu \mathrm{M}$ retinoic acid (RA), followed by 7 days in serum-free DMEM/F12 and 10 $\mathrm{ng} / \mathrm{ml}$ brain-derived neurotrophic factor (BDNF). Note the altered morphology and pronounced neurite outgrowth upon differentiation. Scale bar: $50 \mu \mathrm{m}$. B) Immunostainings of SH-SY5Y-derived neurons (cells were fixed with $3.7 \%$ FA for $1 \mathrm{~h}$, blocked with $5 \%$ BSA and $0.1 \%$ Triton X-100 for 5 minutes, immunostained with polyclonal anti-TAU (K9JA, 1:1000 in PBS, A0024, DAKO, $2^{\text {nd }} \mathrm{AB}$ : donkey anti-rabbit + AlexaFluor488, 1:1000 in PBS, A21202, TFS) and chicken anti-MAP2 (1:2000, ab5392, Abcam, $2^{\text {nd }}$ AB: goat anti-chicken AF647, 1:1000 in PBS, A21449, TFS) antibodies, and mounted (PolyMount, Polysciences), procedure adapted from ${ }^{78}$ ) demonstrate the strong expression and polarized distribution of neuronal maturation markers TAU (green, mainly axonal) and MAP2 (red, mainly somatic). Scale bar (top): $50 \mu \mathrm{m}$, scale bar (bottom): $20 \mu \mathrm{m}$. C) Western blot analysis of TAU isoform expression (de-phosphorylated lysates) in undifferentiated SH-SY5Y cells (lane 1), differently treated SH-SY5Y-derived neurons (lanes 2 - 5) and human brain lysate (lane 6). The very left lane shows a recombinant TAU standard. Note the abundance of all six isoforms upon differentiation with varying ratios compared to the human brain. The blot was adapted and modified from ${ }^{19}$. D) Overview of the TAU protein (grey bar, colored sections indicate distinct TAU domains) and common tauopathy-associated TAU hyperphosphorylation sites (yellow boxes, corresponding epitopes that are recognized by specific antibodies are indicated dashed boxes) that appear highly phosphorylated in SH-SY5Y cells.

Genetic engineering of SH-SY5Y cells. SH-SY5Y-derived neurons display many features of matured neuronal cells, including the post-mitotic character. This makes SH-SY5Y-derived neurons, similarly to primary rodent cultures, inaccessible for stable genetic engineering approaches. However, in the undifferentiated state, SH-SY5Y cells are rapidly dividing and can be used for the stable integration of transgenes, including variants of the TAU-encoding MAPT gene. In the past, transfection and stable integration of linearized 1N3R- and 1N4R-MAPT cDNA into $\mathrm{SH}-\mathrm{SY} 5 \mathrm{Y}$ cells was used to mimic the misbalance of $3 \mathrm{R} / 4 \mathrm{R}$ isoform ratios ${ }^{79,80}$, which is 
caused by MAPT variants that affect alternative splicing in several tauopathies (reviewed in ${ }^{81}$ ). The stable overexpression of $4 \mathrm{R}$ isoforms was also used to assay effects of proteasomal inhibition on TAU protein levels ${ }^{82}$, while integration of a recombinant TAU isoform containing exon 6 unravelled the distinct cellular distribution of this non-canonical TAU isoform similar, which resembles that of MAP2. ${ }^{83}$. By using site-directed recombination assays, recent studies generated SH-SY5Y cells with stable pro-aggregant TAU versions ${ }^{72,84}$. All these approaches have the notable limitation that they are based on artificial overexpression of transgenic TAU isoforms or mutants. The application of recent genome editing techniques, such as CRISPR/Cas9, was shown to work also for SH-SY5Y cells in general ${ }^{85-89}$. The use of CRISPR/Cas9 allows to directly target the endogenous MAPT and generate complete knock-out lines or induce mutations on a single base level that are thought to be involved in TAU (mis)sorting, e.g. by using the improved CRISPR/Cas9 prime editing method ${ }^{90}$.

However, one has to consider the genetic predispositions of SH-SY5Y cells, as large-scale chromosomal abnormalities and imbalances are reported for neuroblastoma cell lines in general 91-95. Accordingly, SH-SY5Y cells show trisomy of chromosome 7 (chr7), a duplication of the qarm of chr1, and further complex rearrangements on the majority of chromosomes leading to both copy number gains and losses ${ }^{96}$. Besides other loci of neurobiological interest, a copy number gain of the MAPT locus on chr17 was confirmed in different studies ${ }^{96-100}$. This genetic arrangement of SH-SY5Y cells complicates the generation of homozygous MAPT mutant knockout (KO) or knock-in cell lines, as it requires editing of presumably three MAPT gene copies, and also impedes heterozygous edits, as it might be difficult to achieve a $50 \%$ decrease of mutant gene expression. However, the successful generation of a MAPT-KO SH-SY5Y cell line recently demonstrated that the SH-SY5Y cells are accessible for CRISPR/Cas9-based homozygous MAPT editing ${ }^{101}$. 


\section{Neuronal identity of SH-SY5Y-derived neurons: Chances and challenges}

The susceptibility to TAU pathology varies drastically among different brain regions, neuronal subtypes and depending on the type of disease ${ }^{102-104}$. Given the fact, that TAU expression, TAU sorting, AIS formation or TAU isoform ratios also differ among neuronal tissues ${ }^{37}$, there might be causal connections between these variations and tissue-specific vulnerability to tauopathies like AD. Therefore, the neuronal identity of a cell model for studies addressing intracellular TAU sorting and localization mechanisms is of certain relevance.

Undifferentiated SH-SY5Y cells are considered immature catecholaminergic neurons since they express markers of immature neurons ${ }^{23,24}$ and key proteins of the catecholaminergic metabolism $14,23,24,105,106$. Interestingly, the reports about the neuronal identity of mature SH-SY5Y-derived neurons vary depending on the substances used for differentiation protocols (Fig. 2A) ${ }^{14}$. The most common and often-used substance, the vitamin A derivative retinoic acid (RA), was shown to elevate the levels of activated choline acetyltransferase, which is typical for cholinergic neurons 23,107,108. However, the cholinergic character of RA-treated cells is under debate, as the expression of noradrenaline 25 and of the vesicular monoamine transporter, a key enzyme of catecholaminergic neurons, was reported in some studies ${ }^{23,107}$, but not in others ${ }^{17}$. Another common procedure, the combinatorial treatment of RA and the brain-derived neurotrophic factor (BDNF) results in extensively branched neurons, which are categorized based on the expression of marker proteins either as noradrenergic ${ }^{17}$, dopaminergic ${ }^{109}$ or cholinergic ${ }^{110}$. Besides RA and BDNF, phorbol esters (e.g. Phorbol-12-myristate-13-acetate (TPA)) ${ }^{25,111-113}$, dibutyryl-cAMP (dbCAMP) ${ }^{114-116}$ or other drugs are used alone or in combination ${ }^{25,117}$ to generate SH-SY5Y-derived neurons with varying neuronal identity, e.g. noradrenergic (TPA, db-cAMP) or dopaminergic (RA+TPA).

Taken together, the classification of SH-SY5Y-derived neurons may depend on the applied substances and be influenced by the focuses of the actual study. It is, however, undisputed that SH-SY5Y-derived neurons resemble key features of noradrenergic, dopaminergic and cholinergic neurons. This gives rise to both i) the great potential of SH-SY5Y-derived neurons for studies on neuronal subtype-specific AD/tauopathy susceptibility and ii) the accompanying challenges, as summarized and discussed below (Fig. 2B). 
Chances. Neuronal loss occurs within several subcortical nuclei in AD patients' brains and, depending on the nuclei, already in early disease stages. These subcortical nuclei are, amongst others, the Locus coeruleus (LC, harbouring noradrenergic neurons), the Nucleus basalis (NB, cholinergic neurons) and the Substantia nigra (SN, dopaminergic neurons) (reviewed in ${ }^{118}$ ). TAUNFT formation in NB neurons and massive depletion of acetylcholine within cortical and hippocampal regions, resulting from loss of NB cholinergic projections, coincide with early clinical symptoms of $A D^{118-124}$. Also the noradrenergic neurons of the $L C$ complex are early affected by NFT formation and degeneration in AD patients ${ }^{125-130}$, and seem to become compromised even in young adults without any clinical phenotype ${ }^{103}$. Within the SN, TAU-NFT formation, pigmented neuronal loss and other pathological alterations are found in AD ${ }^{131-136}$ and other tauopathies 137,138 .

While comprehensive descriptions of TAU-NFT formation and neuronal loss in these subcortical nuclei are available, the pathomechanisms underlying their vulnerability are elusive ${ }^{139}$. If $\mathrm{SH}$ SY5Y-derived neurons properly resemble the neuronal subtypes of LC, NB or SN neurons, they may be a powerful tool for the evaluation of subtype-dependent vulnerability. As described above, several aspects mimicking neuronal TAU physiology are available in SH-SH5Y-derived neurons: subcellular distribution, phosphorylation state, isoform expression levels and ratios, isoformspecific intracellular localization of TAU and cell-stress induced development of NFT formation or, at least, NFT-like hyperphosphorylation.

Challenges. As for all cellular models of $A D$ or related tauopathies, which are largely ageingdependent disorders ${ }^{140-143}$, it is questionable whether up to three-week-old neuronal cultures can resemble the cellular properties of subcortical neurons in the brain of aged $A D$ patients. Furthermore, there are the mentioned inconsistencies of expression profiles, e.g. of RA- and RA/BDNF-treated cells, and it remains uncertain whether the neuronal subtype can be clearly defined. Especially, this is a considerable issue, since a more comprehensive biochemical characterization of the generated neurons would be expensive and time-consuming, without the guarantee of a conclusive outcome. Indeed, the available data rather suggest that SH-SY5Yderived neurons do not resemble clearly segregated and distinct neuronal subtypes, which can be separated by protein expression or transmitter release, but rather exhibit different manifestations of a gradual neuronal entity.

The biggest obstacle of using SH-SY5Y-derived neurons may be that major features of LC, NB and $\mathrm{SN}$ neurons, which may contribute to their susceptibility for early TAU pathology in disease, 
appear to be difficult to recapitulate in cell culture. For LC neurons (reviewed in ${ }^{139}$ ), these features include i) the up to several cm-long, thin and poorly myelinated, heavily branched axons spanning throughout the cortex without relay, which leads to high energy costs and oxidative stress ${ }^{144-146}$ (SH-SY5Y-derived neurons: axons range roughly between 50-150 $\mu \mathrm{m}$ for RA, TPA or db-cAMP treatment 107,111,114,117 and up $200 \mu \mathrm{m}$ and more for RA/BDNF 17,20, and show only moderate branching), ii) increased energy demand and ROS production due to the tonic activity ${ }^{147}$ ( $\mathrm{SH}$ SY5Y-derived neurons: increased excitability and membrane potentials ${ }^{148-150}$ but no tonic activity) and iii) elevated exposure to toxins and pathogens as LC neurons innervate the CNS capillary system and associated astrocyte end feet ${ }^{151-153}$. As all subcortical nuclei share great similarities regarding morphology and innervation ${ }^{154,155}$, the mentioned risk factors may be largely true for NB and SN neurons, as well ${ }^{144,145,156}$.

The targeted differentiation of SH-SY5Y cells into neurons with features of noradrenergic, dopaminergic or cholinergic neurons bears great potential for research on AD-selective vulnerability since the mimicked subcortical nuclei are early affected in AD patients. However, the generation of distinct neuronal subtypes does not appear clearly defined with current differentiation procedures, and SH-SY5Y-derived neurons lack major characteristics of their in vivo correlates, that might be crucial for tauopathy-related vulnerability. 

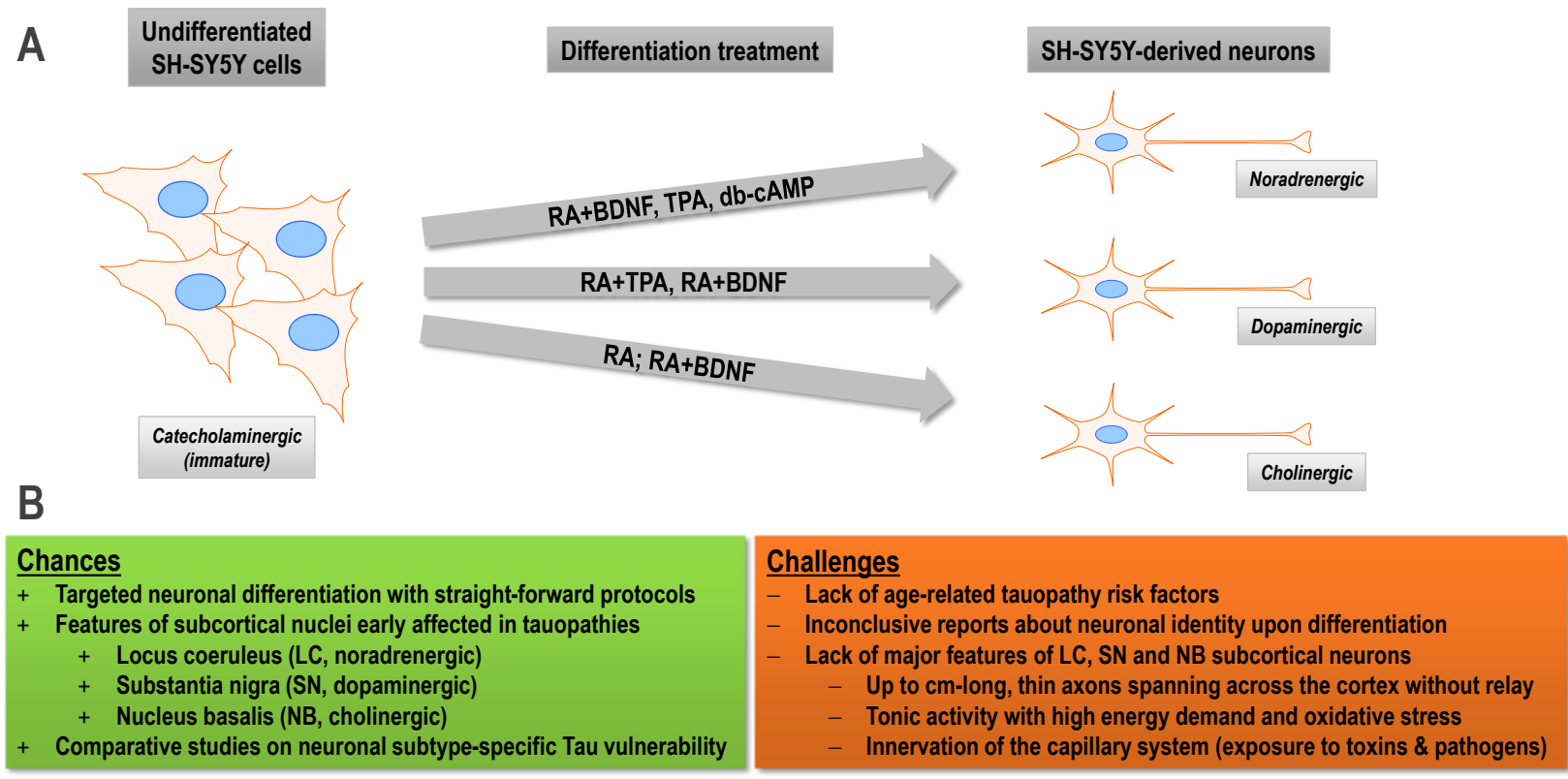

\section{SH-SY5Y-derived neurons}
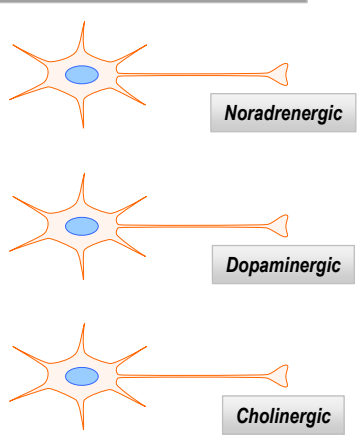

Figure 2: Treatment-dependent neuronal identity of SH-SY5Y-derived neurons. A) Overview of the reported neuronal subtypes for undifferentiated SH-SY5Y cells and SH-SY5Y-derived neurons with respect to commonly used substances (grey arrows) for differentiation. B) Chances (green) and challenges (orange) of the dirigible differentiation of SH-SY5Y-derived neurons and the reported neuronal subtypes. 


\section{Conclusion}

Human-derived SH-SY5Y neuroblastoma cells are robust, cheap, highly proliferative, and can be differentiated into neuronal cells with straightforward protocols. Differentiated SH-SY5Y-derived neurons meet several requirements of an suitable neuronal cell model for TAU and tauopathy basic research: They exhibit i) fast neuronal maturation and polarity, ii) high levels of total TAU protein, iii) expression of all major human isoforms, iv) efficient axonal targeting of TAU protein, and $v$ ) an human brain-like TAU phosphorylation state. Importantly, SH-SY5Y cells are accessible for genetic manipulation, i.e. stable integration of recombinant TAU transgenes and editing of the MAPT locus by means of recent CRISPR/Cas9-based methods prior to neuronal differentiation.

Moreover, SH-SY5Y-derived neurons resemble, depending on the used treatment, neurons of distinct subcortical LC, NB and SN nuclei that are severely affected in AD and other tauopathies. This dirigible differentiation bears great potential for comparative studies of neuron-specific TAU expression patterns, intracellular localization and vulnerability to TAU pathology. However, there are inherent limitations regarding the translatability to human disease, e.g. the lack of agedependent risk factors, the difficulty of defining the exact neuronal subtype or the lack of brainspanning projections (on a cm-scale) leading to high energetic costs and oxidative stress. These caveats have to be taken into account when addressing cell type-specific vulnerability in $\mathrm{SH}$ SY5Y-derived neurons.

Nonetheless, the properties of SH-SY5Y-derived neurons discussed in this review make them a powerful neuronal cell model for investigating the mechanisms of and requirements for axonal TAU sorting under human-like conditions. 


\section{Acknowledgements}

We thank Sarah Bachmann and others for critical manuscript revision. This work was funded by Else-Kröner-Fresenius Stiftung and supported by a doctoral fellowship of the Studienstiftung des deutschen Volkes. The authors declare that they have no competing interests.

\section{List of Abbreviations}

AD, Alzheimer's Disease; AIS, axon initial segment; BDNF, brain-derived neurotrophic factor; CDC2, cell division cycle kinase 2; CDK5, cyclin-dependent kinase 5; CRISPR/Cas9, clustered regularly interspaced short palindromic repeats/CRISPR-associated protein 9; db-cAMP, dibutyryl-cAMP; hiPSC, human-derived induced pluripotent stem cells; LC, locus coeruleus; MAP2, microtubule-associated protein 2; MAPK, mitogen-activated protein kinase; MAPT; microtubule-associated protein TAU-encoding gene; MT, microtubule, NB, nucleus basalis, NFT, neurofibrillary tangles; PP1, protein phosphatase 1; PP2A, protein phosphatase 2A; RA, retinoic acid; Ser, Serine; SN, substantia nigra; Thr, Threonine; TPA, Phorbol-12-myristate-13-acetate. 


\section{References}

1. Korolev, I. O. Alzheimer 's Disease: A Clinical and Basic Science Review. Med. Student Res. J. (2014).

2. Wischik, C. M. et al. Isolation of a fragment of Tau derived from the core of the paired helical filament of Alzheimer disease. Proc. Natl. Acad. Sci. U. S. A. (1988). doi:10.1073/pnas.85.12.4506

3. Brion, J. P., Passareiro, H., Nunez, J. \& Flament-Durand, J. MISE EN EVIDENCE IMMUNOLOGIQUE DE LA PROTEINE TAU AU NIVEAU DES LESIONS DE DEGENERESCENCE NEUROFIBRILLAIRE DE LA MALADIE D'ALZHEIMER. Arch. Biol. (Liege). (1985).

4. Zempel, H. \& Mandelkow, E. Lost after translation: Missorting of Tau protein and consequences for Alzheimer disease. Trends in Neurosciences (2014). doi:10.1016/j.tins.2014.08.004

5. Janke, C. et al. Phylogenetic diversity of the expression of the microtubule-associated protein Tau: Implications for neurodegenerative disorders. Mol. Brain Res. (1999). doi:10.1016/S0169-328X(99)00079-0

6. Muratore, C. R. et al. The familial alzheimer's disease APPV717I mutation alters APP processing and Tau expression in iPSC-derived neurons. Hum. Mol. Genet. (2014). doi:10.1093/hmg/ddu064

7. Verheyen, A. et al. Using human iPSC-derived neurons to model Tau aggregation. PLoS One (2015). doi:10.1371/journal.pone.0146127

8. Silva, M. C. et al. Human iPSC-Derived Neuronal Model of Tau-A152T Frontotemporal Dementia Reveals Tau-Mediated Mechanisms of Neuronal Vulnerability. Stem Cell Reports (2016). doi:10.1016/j.stemcr.2016.08.001

9. Wang, C. et al. Scalable Production of iPSC-Derived Human Neurons to Identify TauLowering Compounds by High-Content Screening. Stem Cell Reports (2017). doi:10.1016/j.stemcr.2017.08.019

10. Sohn, P. D. et al. Pathogenic Tau Impairs Axon Initial Segment Plasticity and Excitability Homeostasis. Neuron (2019). doi:10.1016/j.neuron.2019.08.008

11. Hu, B. Y. et al. Neural differentiation of human induced pluripotent stem cells follows developmental principles but with variable potency. Proc. Natl. Acad. Sci. U. S. A. (2010). doi:10.1073/pnas.0910012107

12. Gunhanlar, N. et al. A simplified protocol for differentiation of electrophysiologically mature neuronal networks from human induced pluripotent stem cells. Mol. Psychiatry (2017). doi:10.1038/mp.2017.56

13. Biedler, J. L. \& Schachner, M. Multiple Neurotransmitter Synthesis by Human Neuroblastoma Cell Lines and Clones. Cancer Res. (1978).

14. Kovalevich, J. \& Langford, D. Considerations for the use of SH-SY5Y neuroblastoma cells in neurobiology. Methods Mol. Biol. (2013). doi:10.1007/978-1-62703-640-5_2

15. Tanapat, P. Neuronal Cell Markers. Mater. Methods (2013). doi:10.13070/mm.en.3.196 
16. Uberti, D., Rizzini, C., Spano, P. F. \& Memo, M. Characterization of Tau proteins in human neuroblastoma SH-SY5Y cell line. Neurosci. Lett. (1997). doi:10.1016/S03043940(97)00715-5

17. Encinas, M. et al. Sequential treatment of SH-SY5Y cells with retinoic acid and brainderived neurotrophic factor gives rise to fully differentiated, neurotrophic factordependent, human neuron-like cells. J. Neurochem. (2000). doi:10.1046/j.14714159.2000.0750991.x

18. Smith, C. J., Anderton, B. H., Davis, D. R. \& Gallo, J. M. Tau isoform expression and phosphorylation state during differentiation of cultured neuronal cells. FEBS Lett. (1995). doi:10.1016/0014-5793(95)01221-Y

19. Agholme, L., Lindström, T., Kgedal, K., Marcusson, J. \& Hallbeck, M. An in vitro model for neuroscience: Differentiation of SH-SY5Y cells into cells with morphological and biochemical characteristics of mature neurons. J. Alzheimer's Dis. (2010). doi:10.3233/JAD-2010-091363

20. Shipley, M. M., Mangold, C. A. \& Szpara, M. L. Differentiation of the SH-SY5Y human neuroblastoma cell line. J. Vis. Exp. (2016). doi:10.3791/53193

21. Goedert, M., Crowther, R. A. \& Garner, C. C. Molecular characterization of microtubuleassociated proteins Tau and map2. Trends in Neurosciences (1991). doi:10.1016/01662236(91)90105-4

22. Cheung, Y. T. et al. Effects of all-trans-retinoic acid on human SH-SY5Y neuroblastoma as in vitro model in neurotoxicity research. Neurotoxicology (2009). doi:10.1016/j.neuro.2008.11.001

23. Lopes, F. M. et al. Comparison between proliferative and neuron-like SH-SY5Y cells as an in vitro model for Parkinson disease studies. Brain Res. (2010). doi:10.1016/j.brainres.2010.03.102

24. Xie, H. R., Hu, L. Sen \& Li, G. Y. SH-SY5Y human neuroblastoma cell line: In vitro cell model of dopaminergic neurons in Parkinson's disease. Chinese Medical Journal (2010). doi:10.3760/cma.j.issn.0366-6999.2010.08.021

25. Påhlman, S., Ruusala, A. I., Abrahamsson, L., Mattsson, M. E. K. \& Esscher, T. Retinoic acid-induced differentiation of cultured human neuroblastoma cells: a comparison with phorbolester-induced differentiation. Cell Differ. (1984). doi:10.1016/0045-6039(84)900381

26. Gimenez-Cassina, A., Lim, F. \& Diaz-Nido, J. Differentiation of a human neuroblastoma into neuron-like cells increases their susceptibility to transduction by herpesviral vectors. J. Neurosci. Res. (2006). doi:10.1002/jnr.20976

27. Rasband, M. N. The axon initial segment and the maintenance of neuronal polarity. Nature Reviews Neuroscience (2010). doi:10.1038/nrn2852

28. Zempel, H. \& Mandelkow, E. Mechanisms of Axonal Sorting of Tau and Influence of the Axon Initial Segment on Tau Cell Polarity. in Advances in Experimental Medicine and Biology (2019). doi:10.1007/978-981-32-9358-8_6

29. Leterrier, C. The axon initial segment: An updated viewpoint. J. Neurosci. (2018). doi:10.1523/JNEUROSCI.1922-17.2018

30. Li, X. et al. Novel diffusion barrier for axonal retention of Tau in neurons and its failure in 
neurodegeneration. EMBO J. (2011). doi:10.1038/emboj.2011.376

31. Zempel, H. et al. Axodendritic sorting and pathological missorting of Tau are isoformspecific and determined by axon initial segment architecture. J. Biol. Chem. (2017). doi:10.1074/jbc.M117.784702

32. Xia, D., Gutmann, J. M. \& Gotz, J. Mobility and subcellular localization of endogenous, gene-edited Tau differs from that of over-expressed human wild-type and P301L mutant Tau. Sci. Rep. (2016). doi:10.1038/srep29074

33. Webster, D. R. \& Borisy, G. G. Microtubules are acetylated in domains that turn over slowly. J. Cell Sci. (1989).

34. Goedert, M., Spillantini, M. G., Jakes, R., Rutherford, D. \& Crowther, R. A. Multiple isoforms of human microtubule-associated protein Tau: sequences and localization in neurofibrillary tangles of Alzheimer's disease. Neuron (1989). doi:10.1016/08966273(89)90210-9

35. Cleveland, D. W., Hwo, S. Y. \& Kirschner, M. W. Physical and chemical properties of purified Tau factor and the role of Tau in microtubule assembly. J. Mol. Biol. (1977). doi:10.1016/0022-2836(77)90214-5

36. Cleveland, D. W., Hwo, S. Y. \& Kirschner, M. W. Purification of Tau, a microtubuleassociated protein that induces assembly of microtubules from purified tubulin. J. Mol. Biol. (1977). doi:10.1016/0022-2836(77)90213-3

37. Goedert, M., Spillantini, M. G., Potier, M. C., Ulrich, J. \& Crowther, R. A. Cloning and sequencing of the cDNA encoding an isoform of microtubule-associated protein Tau containing four tandem repeats: differential expression of Tau protein mRNAs in human brain. EMBO J. (1989). doi:10.1002/j.1460-2075.1989.tb03390.x

38. Nunez, J. \& Fischer, I. Microtubule-Associated Proteins (MAPs) in the Peripheral Nervous System during Development and Regeneration. J. Mol. Neurosci. (1997). doi:10.1007/BF02736834

39. Couchie, D. et al. Primary structure of high molecular weight Tau present in the peripheral nervous system. Proc. Natl. Acad. Sci. U. S. A. (1992). doi:10.1073/pnas.89.10.4378

40. Dupont-Wallois, L. et al. Shift from fetal-type to Alzheimer-type phosphorylated Tau proteins in SKNSH-SY 5Y cells treated with okadaic acid. FEBS Lett. (1995). doi:10.1016/0014-5793(94)01361-4

41. Tanaka, T., Iqbal, K., Trenkner, E., Dong Jie Liu \& Grundke-lqbal, I. Abnormally phosphorylated Tau in SY5Y human neuroblastoma cells. FEBS Lett. (1995). doi:10.1016/0014-5793(95)00061-D

42. Trabzuni, D. et al. MAPT expression and splicing is differentially regulated by brain region: Relation to genotype and implication for Tauopathies. Hum. Mol. Genet. (2012). doi:10.1093/hmg/dds238

43. Goedert, M. \& Jakes, R. Expression of separate isoforms of human Tau protein: correlation with the Tau pattern in brain and effects on tubulin polymerization. EMBO J. (1990). doi:10.1002/j.1460-2075.1990.tb07870.x

44. Spillantini, M. G. \& Goedert, M. Tau protein pathology in neurodegenerative diseases. Trends in Neurosciences (1998). doi:10.1016/S0166-2236(98)01337-X

45. Hong, M. et al. Mutation-specific functional impairments in distinct Tau isoforms of 
hereditary FTDP-17. Science (80-. ). (1998). doi:10.1126/science.282.5395.1914

46. Arendt, T., Stieler, J. T. \& Holzer, M. Tau and Tauopathies. Brain Research Bulletin (2016). doi:10.1016/j.brainresbull.2016.08.018

47. Lee, M., McGeer, E. \& McGeer, P. L. Activated human microglia stimulate neuroblastoma cells to upregulate production of beta amyloid protein and Tau: Implications for Alzheimer's disease pathogenesis. Neurobiol. Aging (2015). doi:10.1016/j.neurobiolaging.2014.07.024

48. He, Z. et al. Transmission of Tauopathy strains is independent of their isoform composition. Nat. Commun. (2020). doi:10.1038/s41467-019-13787-x

49. Hashimoto, S. et al. Tau binding protein CAPON induces Tau aggregation and neurodegeneration. Nat. Commun. (2019). doi:10.1038/s41467-019-10278-x

50. Kovacs, G. G. Invited review: Neuropathology of Tauopathies: Principles and practice. Neuropathology and Applied Neurobiology (2015). doi:10.1111/nan.12208

51. Biernat, J., Gustke, N., Drewes, G., Mandelkow, E. \& Mandelkow, E. Phosphorylation of Ser262 strongly reduces binding of Tau to microtubules: Distinction between PHF-like immunoreactivity and microtubule binding. Neuron (1993). doi:10.1016/08966273(93)90279-Z

52. Morris, M. et al. Tau post-translational modifications in wild-type and human amyloid precursor protein transgenic mice. Nat. Neurosci. (2015). doi:10.1038/nn.4067

53. Usardi, A. et al. Tyrosine phosphorylation of Tau regulates its interactions with Fyn SH2 domains, but not SH3 domains, altering the cellular localization of Tau. FEBS J. (2011). doi:10.1111/j.1742-4658.2011.08218.x

54. Reynolds, C. H. et al. Phosphorylation regulates Tau interactions with Src homology 3 domains of phosphatidylinositol 3-kinase, phospholipase Cy1, Grb2, and Src family kinases. J. Biol. Chem. (2008). doi:10.1074/jbc.M709715200

55. Noble, W., Hanger, D. P., Miller, C. C. J. \& Lovestone, S. The importance of Tau phosphorylation for neurodegenerative diseases. Frontiers in Neurology (2013). doi:10.3389/fneur.2013.00083

56. Kopke, E. et al. Microtubule-associated protein Tau. Abnormal phosphorylation of a nonpaired helical filament pool in Alzheimer disease. J. Biol. Chem. (1993).

57. Zheng-Fischhöfer, Q. et al. Sequential phosphorylation of Tau by glycogen synthase kinase-3 $\beta$ and protein kinase A at Thr212 and Ser214 generates the Alzheimer-specific epitope of antibody AT100 and requires a paired-helical-filament-like conformation. Eur. J. Biochem. (1998). doi:10.1046/j.1432-1327.1998.2520542.x

58. Alavi Naini, S. M. \& Soussi-Yanicostas, N. Tau Hyperphosphorylation and Oxidative Stress, a Critical Vicious Circle in Neurodegenerative Tauopathies? Oxidative Medicine and Cellular Longevity (2015). doi:10.1155/2015/151979

59. Šimić, G. et al. Tau protein hyperphosphorylation and aggregation in alzheimer's disease and other Tauopathies, and possible neuroprotective strategies. Biomolecules (2016). doi:10.3390/biom6010006

60. Buée, L., Bussière, T., Buée-Scherrer, V., Delacourte, A. \& Hof, P. R. Tau protein isoforms, phosphorylation and role in neurodegenerative disorders. Brain Research Reviews (2000). doi:10.1016/S0165-0173(00)00019-9 
61. Hasegawa, M. et al. Characterization of mAb AP422, a novel phosphorylation-dependent monoclonal antibody against Tau protein. FEBS Lett. (1996). doi:10.1016/00145793(96)00271-2

62. Zempel, $\mathrm{H}$. et al. Amyloid- $\beta$ oligomers induce synaptic damage via Tau-dependent microtubule severing by TTLL6 and spastin. EMBO J. (2013). doi:10.1038/emboj.2013.207

63. Majd, S., Koblar, S. \& Power, J. Compound C enhances Tau phosphorylation at Serine396 via PI3K activation in an AMPK and rapamycin independent way in differentiated SH-SY5Y cells. Neurosci. Lett. (2018). doi:10.1016/j.neulet.2018.01.049

64. Tanaka, T., Zhong, J., Iqbal, K., Trenkner, E. \& Grundke-lqbal, I. The regulation of phosphorylation of $\mathrm{T}$ in SY5Y neuroblastoma cells: The role of protein phosphatases. FEBS Lett. (1998). doi:10.1016/S0014-5793(98)00346-9

65. Boban, M., Babić Leko, M., Miškić, T., Hof, P. R. \& Šimić, G. Human neuroblastoma SHSY5Y cells treated with okadaic acid express phosphorylated high molecular weight Tauimmunoreactive protein species. J. Neurosci. Methods (2019). doi:10.1016/j.jneumeth.2018.09.030

66. Sayas, C. L., Moreno-Flores, M. T., Avila, J. \& Wandosell, F. The neurite retraction induced by lysophosphatidic acid increases Alzheimer's disease-like Tau phosphorylation. J. Biol. Chem. (1999). doi:10.1074/jbc.274.52.37046

67. Hernandez, P., Lee, G., Sjoberg, M. \& MacCioni, R. B. Tau phosphorylation by cdk5 and Fyn in response to amyloid peptide A $25-35$ : Involvement of lipid rafts. J. Alzheimer's Dis. (2009). doi:10.3233/JAD-2009-0933

68. Majd, S., Majd, Z., Koblar, S. \& Power, J. Beta estradiol and norepinephrine treatment of differentiated SH-SY5Y cells enhances Tau phosphorylation at (Ser396) and (Ser262) via AMPK but not mTOR signaling pathway. Mol. Cell. Neurosci. (2018). doi:10.1016/j.mcn.2018.02.004

69. Wang, H. Bin, Li, T., Ma, D. Z. \& Zhi, H. ERa36 gene silencing promotes Tau protein phosphorylation, inhibits cell proliferation, and induces apoptosis in human neuroblastoma SH-SY5Y cells. FASEB J. (2018). doi:10.1096/fj.201701386

70. Chan, E. W. L., Krishnansamy, S., Wong, C. \& Gan, S. Y. The NLRP3 inflammasome is involved in the neuroprotective mechanism of neural stem cells against microgliamediated toxicity in SH-SY5Y cells via the attenuation of Tau hyperphosphorylation and amyloidogenesis. Neurotoxicology (2019). doi:10.1016/j.neuro.2018.11.001

71. Yeo, E. T. Y. et al. Piper sarmentosum Roxb. confers neuroprotection on beta-amyloid $(A \beta)$-induced microglia-mediated neuroinflammation and attenuates Tau hyperphosphorylation in SH-SY5Y cells. J. Ethnopharmacol. (2018). doi:10.1016/j.jep.2018.02.025

72. Chen, I. C. et al. Formulated Chinese medicine shaoyao gancao tang reduces Tau aggregation and exerts neuroprotection through anti-oxidation and anti-inflammation. Oxid. Med. Cell. Longev. (2018). doi:10.1155/2018/9595741

73. Nie, S. D. et al. High glucose forces a positive feedback loop connecting ErbB4 expression and mTOR/S6K pathway to aggravate the formation of Tau hyperphosphorylation in differentiated SH-SY5Y cells. Neurobiol. Aging (2018). doi:10.1016/j.neurobiolaging.2018.03.023 
74. Chen, L. et al. Methamphetamine exposure upregulates the amyloid precursor protein and hyperphosphorylated Tau expression: The roles of insulin signaling in sh-sy5y cell line. J. Toxicol. Sci. (2019). doi:10.2131/jts.44.493

75. Olivera-Santa Catalina, M. et al. Hyperosmotic Stress Induces Tau Proteolysis by Caspase-3 Activation in SH-SY5Y Cells. J. Cell. Biochem. (2016). doi:10.1002/jcb.25579

76. Olivera Santa-Catalina, M. et al. JNK signaling pathway regulates sorbitol-induced Tau proteolysis and apoptosis in SH-SY5Y cells by targeting caspase-3. Arch. Biochem. Biophys. (2017). doi:10.1016/j.abb.2017.11.004

77. Liu, Z., Wang, F., Tang, M., Zhao, Y. \& Wang, X. Amyloid $\beta$ and Tau are involved in sleep disorder in Alzheimer's disease by orexin A and adenosine A(1) receptor. Int. J. Mol. Med. (2019). doi:10.3892/ijmm.2018.3935

78. Zempel, H. \& Mandelkow, E. M. Tracking Tau in neurons: How to grow, fix, and stain primary neurons for the investigation of Tau in all developmental stages. in Methods in Molecular Biology (2017). doi:10.1007/978-1-4939-6598-4_20

79. Delobel, P. et al. Stable-Tau Overexpression in Human Neuroblastoma Cells: An Open Door for Explaining Neuronal Death in Tauopathies. in Annals of the New York Academy of Sciences (2003). doi:10.1196/annals.1299.115

80. MAILLIOT, C. et al. Pathological Tau Phenotypes: The Weight of Mutations, Polymorphisms, and Differential Neuronal Vulnerabilities. Ann. N. Y. Acad. Sci. (2006). doi:10.1111/j.1749-6632.2000.tb06911.x

81. Lee, V. M.-Y., Goedert, M. \& Trojanowski, J. Q. Neurodegenerative Tauopathies. Annu. Rev. Neurosci. (2001). doi:10.1146/annurev.neuro.24.1.1121

82. David, D. C. et al. Proteasomal degradation of Tau protein. J. Neurochem. (2002). doi:10.1046/j.1471-4159.2002.01137.x

83. Luo, M. H., Tse, S. W., Memmott, J. \& Andreadis, A. Novel isoforms of Tau that lack the microtubule-binding domain. J. Neurochem. (2004). doi:10.1111/j.14714159.2004.02508.x

84. Chang, K. H. et al. The aqueous extract of Glycyrrhiza inflata can upregulate unfolded protein response-mediated chaperones to reduce Tau misfolding in cell models of Alzheimer's disease. Drug Des. Devel. Ther. (2016). doi:10.2147/DDDT.S96454

85. Kim, H. et al. CRISPR-Cas9 mediated telomere removal leads to mitochondrial stress and protein aggregation. Int. J. Mol. Sci. (2017). doi:10.3390/ijms18102093

86. Bao, L. et al. Depletion of the human ion channel TRPM2 in neuroblastoma demonstrates its key role in cell survival through modulation of mitochondrial reactive oxygen species and bioenergetics. J. Biol. Chem. (2016). doi:10.1074/jbc.M116.747147

87. Kim, S. et al. GBA1 deficiency negatively affects physiological $\alpha$-synuclein tetramers and related multimers. Proc. Natl. Acad. Sci. U. S. A. (2018). doi:10.1073/pnas. 1700465115

88. Xu, Y., Gao, Y. W. \& Yang, Y. SC79 protects dopaminergic neurons from oxidative stress. Oncotarget (2018). doi:10.18632/oncotarget.23538

89. Prasuhn, J., Mårtensson, C. U., Krajka, V., Klein, C. \& Rakovic, A. Genome-edited, THexpressing neuroblastoma cells as a disease model for dopamine-related disorders: A proof-of-concept study on DJ-1-deficient parkinsonism. Front. Cell. Neurosci. (2018). doi:10.3389/fncel.2017.00426 
90. Anzalone, A. V. et al. Search-and-replace genome editing without double-strand breaks or donor DNA. Nature (2019). doi:10.1038/s41586-019-1711-4

91. Ambros, P. F. et al. International consensus for neuroblastoma molecular diagnostics: Report from the International Neuroblastoma Risk Group (INRG) Biology Committee. British Journal of Cancer (2009). doi:10.1038/sj.bjc.6605014

92. George, R. E. et al. Genome-wide analysis of neuroblastomas using high-density single nucleotide polymorphism arrays. PLoS One (2007). doi:10.1371/journal.pone.0000255

93. Stallings, R. L. Origin and functional significance of large-scale chromosomal imbalances in neuroblastoma. Cytogenetic and Genome Research (2007). doi:10.1159/000108291

94. Schleiermacher, G. et al. Accumulation of segmental alterations determines progression in neuroblastoma. J. Clin. Oncol. (2010). doi:10.1200/JCO.2009.26.7955

95. Schleiermacher, G. et al. Segmental chromosomal alterations lead to a higher risk of relapse in infants with MYCN-non-amplified localised unresectable/disseminated neuroblastoma (a SIOPEN collaborative study). Br. J. Cancer (2011). doi:10.1038/bjc.2011.472

96. Yusuf, M., Leung, K., Morris, K. J. \& Volpi, E. V. Comprehensive cytogenomic profile of the in vitro neuronal model SH-SY5Y. Neurogenetics (2013). doi:10.1007/s10048-0120350-9

97. Spengler, B. A., Biedler, J. L. \& Ross, R. A. A corrected karyotype for the SH-SY5Y human neuroblastoma cell line. Cancer Genetics and Cytogenetics (2002). doi:10.1016/S0165-4608(02)00523-X

98. Kryh, H. et al. Comprehensive SNP array study of frequently used neuroblastoma cell lines; copy neutral loss of heterozygosity is common in the cell lines but uncommon in primary tumors. BMC Genomics (2011). doi:10.1186/1471-2164-12-443

99. Cohen, N., Betts, D. R., Rechavi, G., Amariglio, N. \& Trakhtenbrot, L. Clonal expansion and not cell interconversion is the basis for the neuroblast and nonneuronal types of the SK-N-SH neuroblastoma cell line. Cancer Genet. Cytogenet. (2003). doi:10.1016/S01654608(02)00835-X

100. Do, J. H., Kim, I. S., Park, T. K. \& Choi, D. K. Genome-wide examination of chromosomal aberrations in neuroblastoma SH-SY5Y cells by array-based comparative genomic hybridization. Mol. Cells (2007).

101. Sola, M. et al. Tau affects P53 function and cell fate during the DNA damage response. Nat. Commun. Biol. 3, 245 (2020).

102. Braak, H. \& Braak, E. Neuropathological stageing of Alzheimer-related changes. Acta Neuropathologica (1991). doi:10.1007/BF00308809

103. Braak, H. \& Del Tredici, K. The pathological process underlying Alzheimer's disease in individuals under thirty. Acta Neuropathol. (2011). doi:10.1007/s00401-010-0789-4

104. Dickson, D. W., Kouri, N., Murray, M. E. \& Josephs, K. A. Neuropathology of frontotemporal lobar degeneration-Tau (FTLD-Tau). in Journal of Molecular Neuroscience (2011). doi:10.1007/s12031-011-9589-0

105. Oyarce, A. M. \& Fleming, P. J. Multiple forms of human dopamine $\beta$-hydroxylase in SHSY5Y neuroblastoma cells. Arch. Biochem. Biophys. (1991). doi:10.1016/00039861(91)90573-2 
106. Cuende, J., Moreno, S., Bolaños, J. P. \& Almeida, A. Retinoic acid downregulates Rae1 leading to APCCdh1 activation and neuroblastoma SH-SY5Y differentiation. Oncogene (2008). doi:10.1038/sj.onc.1210987

107. Presgraves, S. P., Ahmed, T., Borwege, S. \& Joyce, J. N. Terminally differentiated SHSY5Y cells provide a model system for studying neuroprotective effects of dopamine agonists. Neurotox. Res. (2003). doi:10.1007/BF03033178

108. Adem, A., Mattsson, M. E. K., Nordberg, A. \& Påhlman, S. Muscarinic receptors in human SH-SY5Y neuroblastoma cell line: regulation by phorbol ester and retinoic acid-induced differentiation. Dev. Brain Res. (1987). doi:10.1016/0165-3806(87)90156-8

109. Neuhaus, J. F. G. et al. Catecholamine metabolism drives generation of mitochondrial DNA deletions in dopaminergic neurons. Brain (2014). doi:10.1093/brain/awt291

110. de Medeiros, L. M. et al. Cholinergic Differentiation of Human Neuroblastoma SH-SY5Y Cell Line and Its Potential Use as an In vitro Model for Alzheimer's Disease Studies. Mol. Neurobiol. (2019). doi:10.1007/s12035-019-1605-3

111. Påhlman, S., Odelstad, L., Larsson, E., Grotte, G. \& Nilsson, K. Phenotypic changes of human neuroblastoma cells in culture induced by 12-O-tetradecanoyl-phorbol-13-acetate. Int. J. Cancer (1981). doi:10.1002/ijc.2910280509

112. Murphy, N. P., Ball, S. G. \& Vaughan, P. F. T. Potassium- and Carbachol-Evoked Release of $[3 \mathrm{H}]$ Noradrenaline from Human Neuroblastoma Cells, SH-SY5Y. J. Neurochem. (1991). doi:10.1111/j.1471-4159.1991.tb02085.x

113. Scott, I. G., Åkerman, K. E. O., Heikkilä, J. E., Kaila, K. \& Andersson, L. C. Development of a neural phenotype in differentiating ganglion cell-derived human neuroblastoma cells. J. Cell. Physiol. (1986). doi:10.1002/jcp.1041280221

114. Kume, T. et al. Dibutyryl cyclic AMP induces differentiation of human neuroblastoma SHSY5Y cells into a noradrenergic phenotype. Neurosci. Lett. (2008). doi:10.1016/j.neulet.2008.07.079

115. Sánchez, S. et al. A cAMP-activated pathway, including PKA and PI3K, regulates neuronal differentiation. Neurochem. Int. (2004). doi:10.1016/S0197-0186(03)00150-5

116. Itano, Y., Ito, A., Uehara, T. \& Nomura, Y. Regulation of Bcl-2 Protein Expression in Human Neuroblastoma SH-SY5Y Cells: Positive and Negative Effects of Protein Kinases C and A, Respectively. J. Neurochem. (2002). doi:10.1046/j.1471-4159.1996.67010131.x

117. Simpson, P. B. et al. Retinoic acid-evoked differentiation of neuroblastoma cells predominates over growth factor stimulation: An automated image capture and quantitation approach to neuritogenesis. Anal. Biochem. (2001). doi:10.1006/abio.2001.5346

118. Lyness, S. A., Zarow, C. \& Chui, H. C. Neuron loss in key cholinergic and aminergic nuclei in Alzheimer disease: A meta-analysis. Neurobiology of Aging (2003). doi:10.1016/S0197-4580(02)00057-X

119. Mesulam, M., Shaw, P., Mash, D. \& Weintraub, S. Cholinergic nucleus basalis Tauopathy emerges early in the aging-MCI-AD continuum. Ann. Neurol. (2004). doi:10.1002/ana.20100

120. Sassin, I. et al. Evolution of Alzheimer's disease-related cytoskeletal changes in the basal nucleus of Meynert. Acta Neuropathol. (2000). doi:10.1007/s004019900178 
121. Davies, P. \& Maloney, A. J. F. SELECTIVE LOSS OF CENTRAL CHOLINERGIC NEURONS IN ALZHEIMER'S DISEASE. The Lancet (1976). doi:10.1016/S01406736(76)91936-X

122. Bowen, D. M., Smith, C. B., White, P. \& Davison, A. N. Neurotransmitter-related enzymes and indices of hypoxia in senile dementia and other abiotrophies. Brain (1976).

doi:10.1093/brain/99.3.459

123. Mufson, E. J. et al. Loss of nucleus basalis neurons containing trkA immunoreactivity in individuals with mild cognitive impairment and early Alzheimer's diseases. J. Comp. Neurol. (2000). doi:10.1002/1096-9861(20001106)427:1<19::AID-CNE2>3.0.CO;2-A

124. Mufson, E. J. et al. Loss of basal forebrain p75NTR immunoreactivity in subjects with mild cognitive impairment and Alzheimer's disease. J. Comp. Neurol. (2002).

doi:10.1002/cne.10122

125. Baker, K. G., Törk, I., Hornung, J. P. \& Halasz, P. The human locus coeruleus complex: an immunohistochemical and three dimensional reconstruction study. Exp. Brain Res. (1989). doi:10.1007/BF00274983

126. German, D. C. et al. Disease-specific patterns of locus coeruleus cell loss. Ann. Neurol. (1992). doi:10.1002/ana.410320510

127. Busch, C., Bohl, J. \& Ohm, T. G. Spatial, temporal and numeric analysis of Alzheimer changes in the nucleus coeruleus. Neurobiol. Aging (1997). doi:10.1016/S01974580(97)00035-3

128. Braak, H. \& Del Tredici, K. Alzheimer's disease: Intraneuronal alterations precede insoluble amyloid- $\beta$ formation. Neurobiology of Aging (2004). doi:10.1016/j.neurobiolaging.2003.12.015

129. Aston-Jones, G. \& Cohen, J. D. AN INTEGRATIVE THEORY OF LOCUS COERULEUSNOREPINEPHRINE FUNCTION: Adaptive Gain and Optimal Performance. Annu. Rev. Neurosci. (2005). doi:10.1146/annurev.neuro.28.061604.135709

130. Grudzien, A. et al. Locus coeruleus neurofibrillary degeneration in aging, mild cognitive impairment and early Alzheimer's disease. Neurobiol. Aging (2007).

doi:10.1016/j.neurobiolaging.2006.02.007

131. Gibb, W. R. G., Mountjoy, C. Q., Mann, D. M. A. \& Lees, A. J. The substantia nigra and ventral tegmental area in Alzheimer's disease and Down's syndrome. J. Neurol. Neurosurg. Psychiatry (1989). doi:10.1136/jnnp.52.2.193

132. Burns, J. M., Galvin, J. E., Roe, C. M., Morris, J. C. \& McKeel, D. W. The pathology of the substantia nigra in Alzheimer disease with extrapyramidal signs. Neurology (2005). doi:10.1212/01.WNL.0000158423.05224.7F

133. Tabaton, M., Schenone, A., Romagnoli, P. \& Mancardi, G. L. A quantitative and ultrastructural study of substantia nigra and nucleus centralis superior in Alzheimer's disease. Acta Neuropathol. (1985). doi:10.1007/BF00690198

134. Storga, D., Vrecko, K., Birkmayer, J. G. D. \& Reibnegger, G. Monoaminergic neurotransmitters, their precursors and metabolites in brains of Alzheimer patients. Neurosci. Lett. (1996). doi:10.1016/0304-3940(95)12256-7

135. Ditter, S. M. \& Mirra, S. S. Neuropathologic and clinical features of Parkinson's disease in Alzheimer's disease patients. Neurology (1987). doi:10.1212/wnl.37.5.754 
136. Forstl, H., Levy, R., Burns, A., Luthert, P. \& Cairns, N. Disproportionate loss of noradrenergic and cholinergic neurons as cause of depression in Alzheimer's disease - A hypothesis. Pharmacopsychiatry (1994). doi:10.1055/s-2007-1014267

137. Spillantini, M. G., Bird, T. D. \& Ghetti, B. Frontotemporal Dementia and Parkinsonism Linked to Chromosome 17: A New Group of Tauopathies. Brain Pathol. (2006). doi:10.1111/j.1750-3639.1998.tb00162.x

138. Spillantini, M. G. et al. Familial multiple system Tauopathy with presenile dementia: A disease with abundant neuronal and glial Tau filaments. Proc. Natl. Acad. Sci. U. S. A. (1997). doi:10.1073/pnas.94.8.4113

139. Satoh, A. \& lijima, K. M. Roles of Tau pathology in the locus coeruleus (LC) in ageassociated pathophysiology and Alzheimer's disease pathogenesis: Potential strategies to protect the LC against aging. Brain Research (2019). doi:10.1016/j.brainres.2017.12.027

140. West, M. J., Coleman, P. D., Flood, D. G. \& Troncoso, J. C. Differences in the pattern of hippocampal neuronal loss in normal ageing and Alzheimer's disease. Lancet (1994). doi:10.1016/S0140-6736(94)92338-8

141. Tarantini, S., Tran, C. H. T., Gordon, G. R., Ungvari, Z. \& Csiszar, A. Impaired neurovascular coupling in aging and Alzheimer's disease: Contribution of astrocyte dysfunction and endothelial impairment to cognitive decline. Experimental Gerontology (2017). doi:10.1016/j.exger.2016.11.004

142. Lindemer, E. R., Greve, D. N., Fischl, B. R., Augustinack, J. C. \& Salat, D. H. Regional staging of white matter signal abnormalities in aging and Alzheimer's disease. Neurolmage Clin. (2017). doi:10.1016/j.nicl.2017.01.022

143. Fjell, A. M., McEvoy, L., Holland, D., Dale, A. M. \& Walhovd, K. B. What is normal in normal aging? Effects of aging, amyloid and Alzheimer's disease on the cerebral cortex and the hippocampus. Progress in Neurobiology (2014). doi:10.1016/j.pneurobio.2014.02.004

144. Braak, H. \& Braak, E. Development of Alzheimer-related neurofibrillary changes in the neocortex inversely recapitulates cortical myelogenesis. Acta Neuropathol. (1996). doi:10.1007/s004010050508

145. Braak, H., Rüb, U., Gai, W. P. \& Del Tredici, K. Idiopathic Parkinson's disease: Possible routes by which vulnerable neuronal types may be subject to neuroinvasion by an unknown pathogen. J. Neural Transm. (2003). doi:10.1007/s00702-002-0808-2

146. German, D. C., White, C. L. \& Sparkman, D. R. Alzheimer's disease: Neurofibrillary tangles in nuclei that project to the cerebral cortex. Neuroscience (1987). doi:10.1016/0306-4522(87)90123-0

147. Sanchez-Padilla, J. et al. Mitochondrial oxidant stress in locus coeruleus is regulated by activity and nitric oxide synthase. Nat. Neurosci. (2014). doi:10.1038/nn.3717

148. Åkerman, K. E. O., Scott, I. G. \& Andersson, L. C. Functional differentiation of a human ganglion cell derived neuroblastoma cell line SH-SY5Y induced by a phorbol ester (TPA). Neurochem. Int. (1984). doi:10.1016/0197-0186(84)90029-9

149. Tosetti, P., Taglietti, V. \& Toselli, M. Functional changes in potassium conductances of the human neuroblastoma cell line SH-SY5Y during in vitro differentiation. J. Neurophysiol. (1998). doi:10.1152/jn.1998.79.2.648 
150. Brown, A. M., Riddoch, F. C., Robson, A., Redfern, C. P. F. \& Cheek, T. R. Mechanistic and functional changes in $\mathrm{Ca} 2+$ entry after retinoic acid-induced differentiation of neuroblastoma cells. Biochem. J. (2005). doi:10.1042/BJ20042127

151. Kisler, K., Nelson, A. R., Montagne, A. \& Zlokovic, B. V. Cerebral blood flow regulation and neurovascular dysfunction in Alzheimer disease. Nature Reviews Neuroscience (2017). doi:10.1038/nrn.2017.48

152. Pamphlett, R. Uptake of environmental toxicants by the locus ceruleus: A potential trigger for neurodegenerative, demyelinating and psychiatric disorders. Med. Hypotheses (2014). doi:10.1016/j.mehy.2013.11.016

153. Cohen, Z., Molinatti, G. \& Hamel, E. Astroglial and vascular interactions of noradrenaline terminals in the rat cerebral cortex. J. Cereb. Blood Flow Metab. (1997). doi:10.1097/00004647-199708000-00008

154. Rossor, M. N. Parkinson's disease and Alzheimer's disease as disorders of the isodendritic core. Br. Med. J. (Clin. Res. Ed). (1981). doi:10.1136/bmj.283.6306.1588

155. Ramón-Moliner, E. \& Nauta, W. J. H. The isodendritic core of the brain stem. J. Comp. Neurol. (1966). doi:10.1002/cne.901260301

156. Theofilas, P., Dunlop, S., Heinsen, H. \& Grinberg, L. T. Turning on the light within: Subcortical nuclei of the isodentritic core and their role in Alzheimer's disease pathogenesis. J. Alzheimer's Dis. (2015). doi:10.3233/JAD-142682 